\title{
Equilibrio ácido-base durante la exposición aérea en el molusco bivalvo Perumytilus purpuratus (Lamarck, 1819) (Bivalvia: Mytilidae)
}

\author{
Acid-base balance during air exposure in the bivalve mollusk Perumytilus purpuratus \\ (Lamarck, 1819) (Bivalvia: Mytilidae) \\ Luis A. Montecinos ${ }^{1}$, José A. Cisternaํㅜ, Cristian W. Cáceres ${ }^{1}$ y Gonzalo S. Saldías ${ }^{2}$ \\ ${ }^{1}$ Departamento de Ecología Costera. Facultad de Ciencias. Universidad Católica de la Santísima Concepción. \\ Casilla 297, Concepción, Chile \\ ${ }^{2}$ Departamento de Oceanografía,. Facultad de Ciencias Naturales y Oceanográficas, Universidad de Concepción, \\ Casilla 160-C, Concepción, Chile \\ ccaceres@ucsc.cl
}

\begin{abstract}
For effects of tidal rhythms, intertidal bivalves are exposed to prolonged daily emersion periods during low tide. As a response to air exposure, the mussel Perumytilus purpuratus (Lamarck, 1819) closes its valves and shifts its metabolism to anaerobic pathways by the interruption of the oxygen flux. Consequently, body fluids low their $\mathrm{pH}$ causing the $\mathrm{CaCO}_{3}$ disolution of the shell, which would act as a $\mathrm{pH}$ buffer. In this study, the role of shell $\mathrm{CaCO}_{3}$ in the regulation of internal homeostasis at different periods of aerial exposure was evaluated. We measured the variation of $\mathrm{pH}, \mathrm{Ca}^{2+}$ and $\mathrm{HCO}_{3}{ }^{-}$concentrations in the extrapallial cavity and $\mathrm{Ca}^{+}$ concentration at the shell of $P$. purpuratus individuals from the low and high intertidal zones. The results show that $\mathrm{pH}$ increases in the extrapallial cavity fluids during the emersion period in the laboratory, followed by a decrease of the shell $\mathrm{Ca}^{2+}$. $\mathrm{Ca}^{2+}$ and $\mathrm{HCO}_{3}{ }^{-}$concentrations did not show a significant variation in extrapallial fluids during the aerial exposure periods. Moreover, significant differences were found in the $\mathrm{Ca}^{2+}$ and $\mathrm{HCO}_{3}{ }^{-}$concentrations at the extrapallial fluids between individuals from low and high tidal levels. However, in the majority of measured variables, in spite of acclimation period, the basal levels were different between individuals from the low and high intertidal zones, suggesting physiological differences due to genetic differences among organisms inhabiting both zones.
\end{abstract}

Key words: Anaerobic metabolism, emersion periods, intertidal zone, central Chile

\section{Introducción}

Los animales intermareales habitan un ambiente altamente variable con eventos periódicos extremos como cambios en la temperatura y salinidad producto de las estaciones, exposición aérea, desecación y deficiencia de oxígeno durante las bajas mareas. En esta zona, los organismos particularmente sésiles deben poseer capacidades
Resumen.- Por efecto de los ritmos de marea, los bivalvos intermareales quedan expuestos a prolongados periodos de emersión diarios durante las mareas bajas. Bajo estas condiciones, el mitílido Perumytilus purpuratus (Lamarck, 1819) cierra sus valvas y entra en anaerobiosis por la interrupción del flujo de oxígeno. En esta situación baja el pH de los fluidos corporales provocando la disolución del $\mathrm{CaCO}_{3}$ de las valvas y que actuaría como amortiguador de $\mathrm{pH}$. En este estudio se evaluaron las fluctuaciones de $\mathrm{pH}, \mathrm{Ca}^{2+} \mathrm{y} \mathrm{HCO}_{3}^{-}$en los fluidos de la cavidad extrapaleal y de $\mathrm{Ca}^{2+}$ en las valvas de individuos de $P$. purpuratus provenientes del intermareal bajo y alto, expuestos a periodos progresivos de emersión. Los resultados indican que el $\mathrm{pH}$ en los fluidos de la cavidad extrapaleal aumentó durante el periodo de emersión en laboratorio, lo cual fue seguido por una disminución de la concentración de $\mathrm{Ca}^{2+}$ de las valvas. Las concentraciones de

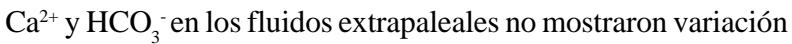
significativa durante el tiempo de exposición aérea. Además, se encontraron diferencias significativas en las concentraciones de $\mathrm{Ca}^{2+} \mathrm{y} \mathrm{HCO}_{3}$ - de los fluidos extrapaleales entre individuos del intermareal rocoso alto y bajo. No obstante lo anterior y a pesar del periodo de aclimatación, los niveles de la mayor parte de las variables medidas presentaron un valor basal diferente entre individuos del intermareal bajo y alto, lo cual sugiere diferencias fisiológicas, debido a diferencias genéticas, entre individuos de ambas zonas.

Palabras clave: Metabolismo anaeróbico, periodos de emersión, zona intermareal, Chile central

fisiológicas que les permitan soportar estas fluctuaciones (Sokolova \& Pörtner 2001, Tomanek \& Helmuth 2002, Almeida \& Bainy 2006). La intensidad del estrés fisiológico para los organismos marinos se incrementa desde el intermareal bajo hacia el intermareal alto (Sokolova \& Pörtner 2001), lo cual afectaría su distribución vertical, ocupando los organismos menos resistentes los niveles mas bajos del intermareal 
(Simpfendörfer et al. 1995, Sepúlveda et al. 2003). Se ha descrito que algunos bivalvos intermareales deben soportar más de 12 h de emersión antes de la siguiente marea alta (Byrne \& McMahon 1991).

Para soportar la emersión, los organismos deben poseer mecanismos de compensación, que les permitan conservar la homeostasis durante la emersión. Estos mecanismos incluyen aspectos conductuales, tales como la abertura de las valvas, y fisiológicos como intercambio de gas aéreo y eficientes vías anaeróbicas (Boyden 1972, Widdows et al. 1979, Byrne et al. 1990). Además se ha descrito la movilización del $\mathrm{CaCO}_{3}$ de las valvas para mantener el balance ácido-base, evitando la acidosis como consecuencia del metabolismo anaeróbico (Byrne \& McMahon 1991) y de esta manera mantener el equilibrio interno.

En condiciones de inmersión, en mareas altas, los bivalvos desarrollan principalmente metabolismo aeróbico, con apertura de valvas e intercambio gaseoso branquial (Crenshaw 1980). En esta situación, el molusco es capaz de bombear activamente material rico en $\mathrm{CaCO}_{3}$ a la cavidad extrapaleal, pudiéndose depositar nuevo material en las valvas en forma de cristales de calcita y/o aragonita dependiendo de la especie (Crenshaw 1980). Por otra parte, bajo condiciones de emersión durante mareas bajas, los bivalvos intermareales cierran sus valvas, desencadenándose metabolismo anaeróbico, produciéndose una acumulación de moléculas inorgánicas, como $\mathrm{CO}_{2}$ en la cavidad extrapaleal, con una disminución del pH y la disolución del $\mathrm{CaCO}_{3}$ que aún no se ha consolidado en las valvas como matriz estructural sólida (Ruppert \& Barnes 1996), empleándose como amortiguador de pH (Byrne \& McMahon 1991).

El bivalvo Perumytilus purpuratus (Lamarck, 1819) es un mitílido que forma mantos en la zona intermareal de las costas de Chile, ocupando gran parte del gradiente vertical (Alvarado \& Castilla 1996), con prolongados periodos de exposición aérea durante las mareas bajas (Guzmán et al. 1998), los cuales varían según la altura del intermareal en que se encuentre el organismo. En este trabajo se compararon experimentalmente las respuestas, en el equilibrio ácido-base, a la emersión en individuos de $P$. purpuratus provenientes de los niveles intermareales alto y bajo.

\section{Material y métodos}

Los especimenes de Perumytilus purpuratus del intermareal bajo y alto fueron recolectados manualmente, mediante espátula, en períodos de marea baja, durante octubre de 2006 en la playa rocosa de Lirquén (3642‘30"S; 7258‘42"W), en la bahía de Concepción, zona centro-sur de Chile. Los individuos recolectados fueron puestos en contenedores con agua de mar y trasladados al laboratorio, donde fueron medidos, pesados y sus valvas limpiadas de epibiontes. Posteriormente, fueron aclimatados en una cámara refrigerada a $13 \pm$ $0,5^{\circ} \mathrm{C}$, con aireación constante, recambio periódico de agua a salinidad de 30 ups e inanición por una semana.

Los ejemplares de Perumytilus purpuratus provenientes de ambas zonas del intermareal fueron expuestos a 7 condiciones experimentales: 0 (inmersión), 2, 4, 6, 8, 10 y 12 h de exposición aérea; para esto, los individuos fueron ubicados individualmente en bandejas plásticas y retirados a cada tiempo experimental. A cada individuo se le extrajo mediante punción, líquido de la cavidad extrapaleal para determinar las concentraciones de metabolitos y su pH (Lagos \& Cáceres 2008).

La medición de $\mathrm{pH}$ de los fluidos extrapaleales se realizó utilizando un pHímetro HANNA modelo 1332. En la cuantificación del contenido de $\mathrm{HCO}_{3}{ }^{-}$en los fluidos extrapaleales, se utilizó el método de determinación titrimética de $\mathrm{HCO}_{3}$ (Henry et al. 1980), utilizando 1,5 $\mathrm{mL}$. A cada muestra se le agregó 1,0 mL de $\mathrm{HCl}$ 0,05 M y se mezcló mediante agitación durante un minuto. Posteriormente se realizó una titulación con $\mathrm{NaOH}$ $(0,1 \mathrm{M})$.

Para medir las concentraciones de $\mathrm{Ca}^{2+}$ de fluidos extrapaleales y valvas de individuos de Perumytilus purpuratus, se utilizó el método colorimétrico de Moorehead \& Biggs (1974), utilizando $10 \mu \mathrm{L}$ de fluido extrapaleal para la determinación de $\mathrm{Ca}^{2+}$ en fluido. Para la determinación de la concentración de $\mathrm{Ca}^{2+}$ en las valvas, se secó y limpió la valva izquierda, la cual fue triturada en un mortero de porcelana; se tomaron $0,5 \mathrm{~g}$ de ella y se le agregaron $2 \mathrm{~mL}$ de $\mathrm{HCl}$ 0,1 M durante $10 \mathrm{~s}$ (Gunthorpe et al. 1990); y leídas en un espectrofotómetro Bausch \& Lomb a $570 \mathrm{~nm}$. La medición de $\mathrm{Ca}^{2+}$ en las valvas fue realizada cada cuatro horas.

\section{Análisis estadísticos}

Las diferencias en el tamaño corporal de individuos de Perumytilus purpuratus recolectados en el intermareal bajo y alto fueron evaluadas mediante la prueba $t$ de Student (Zar 1996).

Para analizar los efectos de la exposición aérea sobre las variables estudiadas se utilizó un análisis de varianza (ANDEVA) de dos vías, con especie y tiempo como factores fijos (Zar 1996); la homogeneidad de varianza fue analizada mediante la prueba de Levene; los datos no homogéneos fueron transformados logarítmicamente (Zar 1996). Las diferencias a posteriori se evaluaron utilizando la prueba de Tukey con un nivel de significancia de 0,05. 
Para determinar el grado de relación entre las variables medidas se utilizó un análisis de correlación de Pearson ocupando el total de individuos de la muestra (Zar 1996). Los datos se expresan en el texto como promedio \pm error estándar (x \pm ee).

\section{Resultados}

El peso promedio ( \pm error estándar) de los individuos de Perumytilus purpuratus fue de 7,05 $\pm 0,13 \mathrm{~g}(\mathrm{~N}=70)$ para los organismos recolectados en el intermareal bajo y de 7,10 $\pm 0,16 \mathrm{~g}(\mathrm{~N}=70)$ para los individuos recolectados en el intermareal alto, no existiendo diferencias en el tamaño de los individuos recolectados en el intermareal bajo y alto (Student-t, $\mathrm{t}_{(1,138)}=-0,02, P=0,99$ ).

\section{pH de fluidos extrapaleales}

El pH de los fluidos extrapaleales fluctuó entre 6 y 8, existiendo variaciones significativas en función de las dos variables estudiadas y su interacción (intermareal alto y bajo y el tiempo de emersión). Los individuos provenientes del intermareal bajo presentaron un $\mathrm{pH}$ de $7,08 \pm 0,04$ significativamente menor que el de los organismos del intermareal alto, 7,22 \pm 0,02 (Tabla 1). En el factor tiempo, el pH de 6,92 \pm 0,08 obtenido a las dos horas de exposición aérea fue significativamente menor que el valor de 7,38 \pm 0,05 observado a las $12 \mathrm{~h}$ de exposición (Fig. 1A). La interacción entre altura del intermareal y tiempo se observó en los individuos del intermareal bajo, los cuales presentaron un $\mathrm{pH}$ menor a las 2 h de exposición, 6,63 $\pm 0,08$, y el máximo $\mathrm{pH}$ de $7,42 \pm 0,10$ a las 12 h de exposición aérea.

\section{Concentración de $\mathrm{Ca}^{2+}$ y $\mathrm{HCO}_{3}^{-}$en fluidos extrapaleales}

En la concentración de $\mathrm{HCO}_{3}^{-}$de los fluidos extrapaleales de Perumytilus purpuratus solo se observó un efecto significativo del nivel intermareal, siendo la concentración de 31,32 \pm 0,23 mmol L ${ }^{-1}$ obtenida en los organismos recolectados en el intermareal bajo significativamente mayor que la medida en los organismos del intermareal alto, la cual fue de 17,86 $\pm 0,95 \mathrm{mmol} \mathrm{L}^{-1}$ (Tabla 1, Fig. 1B).

Del mismo modo, la concentración de $\mathrm{Ca}^{2+}$ en los fluidos extrapaleales de los organismos recolectados del intermareal bajo durante las $12 \mathrm{~h}$ de experimentación fue de 2,71 \pm 0,05 mmol L-1 significativamente mayor que la concentración de 2,21 $\pm 0,03 \mathrm{mmol} \mathrm{L}^{-1}$ medida en los organismos del intermareal alto (Tabla 1, Fig. 1C). En la concentración de $\mathrm{HCO}_{3}$ no se observó un efecto significativo del tiempo o de la interacción entre tiempo y nivel del intermareal.

\section{Concentración de $\mathrm{Ca}^{2+}$ en las valvas}

Tanto para los individuos de Perumytilus purpuratus provenientes del intermareal bajo como los del intermareal alto, la concentración de $\mathrm{Ca}^{2+}$ en las valvas disminuyó significativamente con el tiempo de exposición aérea, con un mínimo de 3,42 \pm 0,09 mmol L-1 a las $12 \mathrm{~h}$ de exposición aérea y un valor máximo de 3,82 \pm 0,06 $\mathrm{mmol} \mathrm{L}^{-1}$ en los individuos en inmersión (tiempo 0) (Fig. 1D). No se encontraron diferencias significativas entre los organismos provenientes de ambos niveles ni en la interacción entre nivel mareal y tiempo de exposición aérea (Tabla 1).

\section{Tabla 1}

Análisis de varianza de dos vías para los niveles de $\mathrm{pH}, \mathrm{Ca}^{2+}$ y $\mathrm{HCO}_{3}^{-}$de fluidos extrapaleales y $\mathrm{Ca}^{2+}$ de las valvas de individuos de Perumytilus purpuratus provenientes de dos niveles intermareales (alto y bajo) y expuestos a distintos tiempos de emersión (GL=grados de libertad, $\mathbf{C M}=$ cuadrados medios, $F=$ valor de $F, P=$ probabilidad)

Two-way analysis of variance for the levels of $\mathrm{pH}, \mathrm{Ca}^{2+}{\mathrm{y} \mathrm{HCO}_{3}}^{-}$from extrapallial fluids and $\mathrm{Ca}^{2+}$ from the valves of Perumytilus purpuratus individuals collected from two intertidal levels (high and low) under different air exposure times (GL=degrees of freedom, $\mathrm{CM}=$ means square, $\mathrm{F}=\mathrm{F}$ value, $P=$ =probability)

\begin{tabular}{|c|c|c|c|c|c|c|c|c|c|c|c|c|c|c|c|c|}
\hline & \multicolumn{4}{|c|}{$\mathrm{pH}$} & \multicolumn{4}{|c|}{$\mathrm{HCO}_{3}^{-}$} & \multicolumn{4}{|c|}{$\begin{array}{c}\mathrm{Ca}^{2+} \\
\text { Fluidos extrapaleales }\end{array}$} & \multicolumn{4}{|c|}{$\begin{array}{c}\mathrm{Ca}^{+} \\
\text {Concha }\end{array}$} \\
\hline & GL & $\mathrm{CM}$ & $\mathrm{F}$ & $P$ & GL & $\mathrm{CM}$ & $\mathrm{F}$ & $P$ & GL & $\mathrm{CM}$ & $\mathrm{F}$ & $P$ & GL & $\mathrm{CM}$ & $\mathrm{F}$ & $P$ \\
\hline Nivel Mareal & 1 & 0,002 & 14,49 & $<0,01$ & 1 & 2,865 & 141,56 & $<0,01$ & 1 & 0,24 & 61,34 & $<0,01$ & 1 & 0,008 & 0,49 & 0,48 \\
\hline Tiempo de exposición & 6 & 0,001 & 10,11 & $<0,01$ & 6 & 0,012 & 0,60 & 0,72 & 6 & 0,007 & 1,91 & 0,08 & 3 & 0,011 & 6,73 & $<0,01$ \\
\hline Interacción & 6 & 0,0009 & 5,27 & $<0,01$ & 6 & 0,001 & 0,34 & 0,91 & 6 & 0,002 & 0,50 & 0,80 & 3 & 0,002 & 1,04 & 0,38 \\
\hline Error & 126 & 0,0002 & & & 126 & 0,020 & & & 126 & 0,004 & & & 72 & 0,002 & & \\
\hline
\end{tabular}



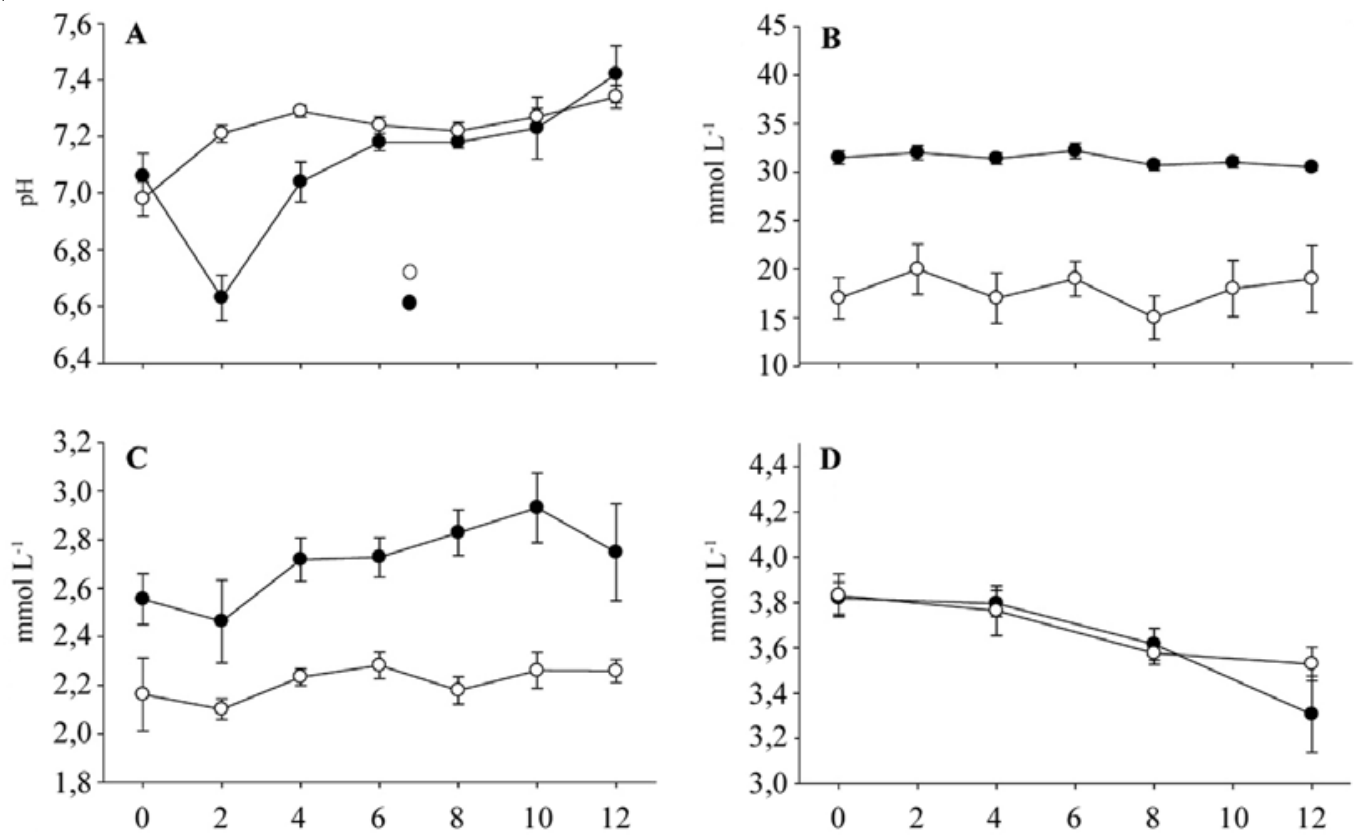

Tiempo de emersión (h)

Intermareal alto

Intermareal bajo

\section{Figura 1}

Concentraciones medidas en Perumytilus purpuratus a distintos tiempos de exposición aérea (x \pm error estándar).

A = Variaciones de pH en fluido extrapaleal ; $\mathrm{B}$ = Concentración de $\mathrm{HCO}_{3}{ }^{-}$en fluido extrapaleal;

$\mathrm{C}=$ Concentración de calcio en fluido extrapaleal; $\mathbf{D}=$ Concentración de calcio en concha.

$$
\text { ( } N=10 \text { en todos los casos) }
$$

Concentrations measured in Perumytilus purpuratus over several times of aerial exposure ( \pm standard error).

$\mathrm{A}=$ Variations in extrapallial $\mathrm{pH} ; \mathrm{B}=\mathrm{HCO}_{3}{ }^{-}$extrapallial fluid concentrations;

$\mathrm{C}=$ Calcium extrapallial fluids concentration; $\mathrm{D}=$ Calcium concentration in shell.

$$
(\mathrm{N}=10 \text { in all cases) }
$$

\section{Tabla 2}

Valores de correlación de Spearman entre las concentraciones medidas en Perumytilus purpuratus $(P=$ probabilidad, Valv=valvas, $\mathrm{Ext}=$ =fluido extrapalaeal)

Spearman correlation values between measured concentrations in

\begin{tabular}{|c|c|c|c|c|}
\hline Variables & $\mathrm{N}$ & Spearman (rs) & $\mathrm{t}(\mathrm{N}-2)$ & $P$ \\
\hline$\left[\mathrm{HCO}_{3}{ }^{-}\right]$Ext y $\left[\mathrm{Ca}^{2+}\right]$ Ext & 140 & 0,51 & 7,05 & $<0,001$ \\
\hline $\mathrm{pH}$ y $\left[\mathrm{HCO}_{3}{ }^{-}\right] \mathrm{Ext}$ & 140 & $-0,19$ & $-2,36$ & $<0,05$ \\
\hline$\left[\mathrm{Ca}^{2+}\right]$ Ext y y $\left[\mathrm{Ca}^{2+}\right]$ Valv & 80 & $-0,23$ & $-2,12$ & $<0,05$ \\
\hline $\mathrm{pH}$ y $\left[\mathrm{Ca}^{2+}\right]$ Valv & 80 & $-0,17$ & $-1,59$ & 0,11 \\
\hline $\mathrm{pH}$ y $\left[\mathrm{Ca}^{2+}\right]$ Ext & 140 & $-0,12$ & $-1,43$ & 0,15 \\
\hline$\left[\mathrm{HCO}_{3}{ }^{-}\right]$Ext y $\left[\mathrm{Ca}^{2+}\right]$ Valv & 80 & 0,032 & 0,28 & 0,78 \\
\hline
\end{tabular}
Perumytilus purpuratus ( $P=$ probability value, Valv=shell, Ext=extrapallial fluid) 
Se encontraron correlaciones positivas y significativas entre: la concentración de $\mathrm{HCO}_{3}^{-} \mathrm{y} \mathrm{Ca}^{2+}$ en los fluidos extrapaleales (rs $=0,51, P<0,01$ ) (Tabla 2). Por otra parte se observaron relaciones negativas y significativas entre: el $\mathrm{pH}$ y la concentración de $\mathrm{HCO}_{3}^{-}$en los fluidos extrapaleales (rs $=-0,19, P<0,05$ ) y la concentración de $\mathrm{Ca}^{2+}$ en las valvas y el $\mathrm{Ca}^{2+}$ en el fluido extrapaleal (rs = 0,23, $P<0,05$ ) (Tabla 2).

\section{Discusión}

En este estudio se ha demostrado que Perumytilus purpuratus tiene una capacidad diferencial para tolerar fisiológicamente el efecto de la exposición aérea asociada a la zona del intermareal de la cual provienen los organismos y al tiempo en que están expuesto al aire cuando ocurren las bajas mareas.

Se ha descrito que moluscos bivalvos que habitan en niveles intermareales más altos presentan una mayor afinidad terrestre en comparación con individuos del nivel intermareal bajo, que presentarían mayor afinidad marina (Hummel et al. 1988, Simpfendörfer et al. 1995). Sin embargo, son pocos los trabajos que tratan comparativamente sobre organismos que habitan toda la franja del intermareal.

El aumento en el pH de la cavidad extrapaleal de los individuos de $P$. purpuratus de ambos niveles intermareales durante la emersión (Fig. 1A), se debe probablemente al desarrollo de metabolismo anaeróbico por la falta de oxígeno disponible en forma disuelta para el intercambio branquial (Stickle et al. 1989, Ruppert \& Barnes 1996). Esto genera un aumento de metabolitos ácidos en los fluidos extrapaleales como el $\mathrm{H}_{2} \mathrm{CO}_{3}$ (Tabla 2), motivo por el cual se produce la disolución del $\mathrm{CaCO}_{3}$ que aún no se ha consolidado como parte de las valvas y que actúa como un amortiguador de $\mathrm{pH}$ para disminuir la acidez en los fluidos de dicha cavidad (Creenshaw 1980). Por otro lado, también se ha reportado al $\mathrm{CaCO}_{3}$ como fuente amortiguadora de $\mathrm{pH}$ en la hemolinfa de moluscos bivalvos y crustáceos (Henry et al. 1981, Lagos \& Cáceres 2008).

En este estudio se observó que durante la emersión experimental, el $\mathrm{pH}$ de la cavidad extrapaleal no se mantuvo constante, sino que aumentó en los individuos del intermareal bajo como alto (Fig. 1A), lo cual puede significar la expresión de un mecanismo de sobrecompensación por la mayor liberación de $\mathrm{CO}_{2}$ producto de la anaerobiosis, lo cual tendería a generar acidificación de los fluidos corporales, fenómeno observado en otras especies de bivalvos (Byrne \& McMahon 1991). En el caso de $P$. purpuratus, esta sobrecompensación podría ser producto de la liberación de $\mathrm{CaCO}_{3}$ de las valvas, la cual induce una basificación de los fluidos, lo que se refuerza con el hecho que esta especie presenta una gruesa concha (Guzmán et al. 1998), la cual puede ser considerada una abundante fuente de $\mathrm{CaCO}_{3}$. Además, se observaron diferencias en la variación de $\mathrm{pH}$ entre individuos de distinto nivel intermareal; esto puede ser interpretado como la expresión de una característica con fuerte base genética, porque si fuera solo producto de la plasticidad fenotípica, el período de aclimatación previo debería haber uniformado las respuestas. Esto se refuerza con la existencia de interacción entre ambos factores (nivel intermareal y tiempo de emersión) en el cual los valores extremos se dan en el intermareal bajo a las 2 y 12 h de emersión, lo cual indica una incapacidad de respuesta a corto (2 h) y largo plazo (12 h) en estos organismos, a diferencia de lo observado en los provenientes del intermareal alto.

El carbonato de calcio depositado en las valvas de los moluscos bivalvos, así como en el exoesqueleto de otros organismos intermareales como crustáceos (Cameron 1990, Tomanek \& Helmuth 2002), es considerada la principal fuente de calcio para los organismos (Henry et al. 1981). Mangum et al. (1979) observaron un incremento en $\mathrm{Ca}^{2+}{\mathrm{y} \mathrm{HCO}_{3}}^{-}$durante el cierre de las valvas del molusco bivalvo Rangia cuneata producto del metabolismo anaeróbico. Estos moluscos de respiración acuática pueden utilizar el carbonato de calcio de las valvas durante largos periodos en que éstas se encuentran cerradas (i.e. exposición aérea), debido al corte en el transporte de iones en el ambiente submarino (Henry et al. 1981). En organismos como el cangrejo azul Callinectes sapidus, se ha documentado que el exoesqueleto está compuesto tanto por una matriz insoluble y otra soluble ante la disminución del pH para regular el equilibrio ácido-base interno (Gunthorpe et al. 1990). En la concentración de calcio en los fluidos de la cavidad extrapaleal se encontraron diferencias significativas entre individuos de distintos niveles intermareales, lo que refuerza los posibles contrastes en adaptación al medio aéreo expuestos en el punto anterior. Esta falta de cambios en función de la emersión en esta especie contrasta con lo encontrado en otros organismos como bivalvos (Byrne \& McMahon 1991) y crustáceos (Lagos \& Cáceres 2008); esta falta de cambios podría deberse a la movilización de $\mathrm{Ca}^{2+}$ a la hemolinfa para ser eventualmente utilizado como agente osmótico, disminuyendo de esta manera la pérdida de agua.

Estudios realizados con el molusco bivalvo Anodonta grandis simpsoniana reportan aumento del contenido de $\mathrm{HCO}_{3}$ - de los fluidos extracelulares durante un prolongado período de exposición al aire (Byrne \& McMahon 1991). Un patrón similar ocurrió para Rangia cuneata (Mangum 
et al. 1979), que tuvo un aumento en la concentración de $\mathrm{Ca}^{2+}$ y $\mathrm{HCO}_{3}^{-}$durante el cierre de las valvas como consecuencia del metabolismo anaerobio. En $P$. purpuratus, no se observó un notable aumento en la concentración de $\mathrm{HCO}_{3}^{-}$de los fluidos extrapaleales durante el periodo de exposición aérea (Fig. 1B). Por otro lado, solo se encontraron diferencias significativas entre los dos niveles mareales al igual que con la concentración de $\mathrm{Ca}^{2+}$ (Fig. 1C), lo que confirma lo señalado anteriormente en el sentido una capacidad fisiológica mayor en individuos provenientes del intermareal alto para soportar el impacto diferencial de los factores físicos en el gradiente vertical (Simpfendörfer et al. 1995).

La disminución en la concentración de $\mathrm{Ca}^{2+}$ de las valvas en los distintos tiempos de exposición aérea para los individuos de ambos niveles mareales (Fig. 1D), demostraría el transporte de iones desde las valvas hacia los fluidos extrapaleales para mantener el equilibrio ácidobase como indica el análisis de correlación con una relación significativa y negativa entre la concentración de calcio de los fluidos y de la concha. No obstante, la falta de diferencias significativas entre los niveles mareales (al igual que en la concentración de $\mathrm{Ca}^{2+} \mathrm{y} \mathrm{HCO}_{3}^{-}$ de los fluidos extrapaleales en los tiempos de exposición aérea) se debería a la existencia de otros iones de potencial importancia en la regulación ácido-base, como se ha descrito con el $\mathrm{Na}^{+}$y Cl$^{-}$(Byrne \& Dietz 1997).

Según los resultados encontrados en este estudio, Perumytilus purpuratus tiene una clara adaptación a la vida en el intermareal, ya que muestra tolerancia a la exposición aérea durante tiempos de emersión prolongados, contrarrestando la disminución del $\mathrm{pH}$ en sus fluidos extrapaleales con la remoción de material cálcico desde sus valvas. Estas observaciones fueron más evidentes en individuos del nivel intermareal alto, los cuales tendrían una mayor adaptación a condiciones de exposición aérea por mostrar una rápida respuesta en el control del aumento de la acidez en los fluidos extrapaleales a lo largo del experimento.

Los escasos estudios sobre respuestas fisiológicas de moluscos marinos ante la heterogeneidad del sistema costero dejan de manifiesto una ventana necesaria de investigar, considerando la marcada variabilidad temporal de las condiciones físicas del sistema litoral, lo que afecta no sólo la distribución de los organismos sino que también su diversidad.

\section{Agradecimientos}

Se agradece a los revisores anónimos por sus comentarios y sugerencias. Este trabajo forma parte de la Tesis de
Grado presentada por L. Montecinos para optar al titulo profesional de Biólogo Marino de la Universidad Católica de la Ssma. Concepción.

\section{Literatura citada}

Almeida EA \& ACD Bainy. 2006. Effect of aerial exposure on antioxidant defenses in the brown mussel Perna perna. Brazilian Archives of Biology and Technology 49: 225229.

Alvarado JL \& JC Castilla. 1996. Tridimensional matrices of Perumytilus purpuratus on intertidal platforms with varying wave forces in central Chile. Marine Ecology Progress Series 133: 135-141.

Boyden CR. 1972. Aerial respiration in the cockle Cerastoderma edule in relation to temperature. Comparative Biochemistry and Physiology 43: 697-712.

Byrne R, E Gnaiger, R McMahon \& T Dietz. 1990. Behavioral and metabolic responses to emersion and subsequent reimmersion in the freshwater bivalve Corbicula fluminea. The Biological Bulletin 178: 251-259.

Byrne R \& R McMahon. 1991. Acid-base and ionic regulation, during and following emersion, in the freshwater bivalve, Anodonta grandis simpsoniana (Bivalvia: Unionidae). The Biological Bulletin 181: 289-297.

Byrne R \& T Dietz. 1997. Ion transport and acid-base balance in freshwater bivalves. Journal of Experimental Biology 200: 457-465.

Cameron J. 1990. Unusual aspects of calcium metabolism in aquatic animals. Annual Review of Physiology 52: 77-95.

Crenshaw MA. 1980. Mechanisms of shell formation and dissolution. En: Rhoads D \& R. Lutz (eds). Skeletal growth of aquatic organisms, pp. 115-132. Plenum Press, New York.

Gunthorpe E, C Sikes \& A Wheeler. 1990. Promotion and inhibition of calcium carbonate crystallization in vitro by matrix protein from blue crab exoskeleton. The Biological Bulletin 179: 191-200.

Guzmán N, S Sheyla \& L Ortlieb. 1998. Catálogo descriptivos de los moluscos litorales (Gastropoda y Pelecypoda) de la zona de Antofagasta, $23^{\circ} \mathrm{S}$ (Chile). Estudios Oceanológicos 17: 17-86.

Henry R, D Cannon \& J Winkelman. 1980. Química clínica: Bases y técnicas, 819 pp. Editorial Jims, Barcelona.

Henry R, G Kormanik, N Smatresk \& J Cameron. 1981. The role of $\mathrm{CaCO}_{3}$ dissolution as a source of $\mathrm{HCO}_{3}^{-}$for the buffering of hypercapnic acidosis in the aquatic and terrestrial decapod crustaceans. Journal of Experimental Biology 94: 269-274.

Hummel H, A Fortuin, L De Wolf \& A Meijboom. 1988. Mortality of intertidal benthic animals after a period of prolonged emersion. Journal Experimental Marine Biology and Ecology 121: 247-254. 
Lagos M \& C Cáceres. 2008. Como afecta la exposición aérea el equilibrio ácido base de organismos móviles del intermareal: Petrolisthes laevigatus/ (Guérin, 1835) (Decapoda: Porcellanidae), como caso de estudio. Revista de Biología Marina y Oceanografía 43: 591-598

Mangum C, R Henry \& D Simpson. 1979. The effect of ouabain on blood $\mathrm{NaCl}$ in the osmoregulating clam Rangia cuneata. Journal of Experimental Zoology 207: 329-335.

Moorehead W \& H Biggs. 1974. 2-Amino-2-methyl-1propanol as the alkalizing agent in an improve continuousflow cresolphthalein complexone procedure for calcium in serum. Clinical Chemistry 20: 1458-1480.

Ruppert E \& R Barnes. 1996. Zoología de los invertebrados, 1114 pp. McGraw-Hill Interamericana, México.

Sepúlveda R, R Moreno \& F Carrasco. 2003. Diversidad de macroinvertebrados asociados a arrecifes de Phragmatopoma moerchi (Kingbert, 1867) (Polychaeta: Sabellariidae) en el intermareal rocoso de Cocholgüe, Chile. Gayana 67: 45-54.

Simpfendörfer R, V Vial, A López, M Verdala \& L González 1995. Relationship between the aerobic and anaerobic metabolic capacities and the vertical distribution of three intertidal sessile invertebrates: Jehlius cirratus (Darwin) (Cirripedia), Perumytilus purpuratus (Lamarck) (Bivalvia) and Mytilus chilensis (Hupé) (Bivalvia). Comparative Biochemistry and Physiology 111 B. 4: 615-623.

Sokolova M \& H Pörtner. 2001. Physiological adaptations to high intertidal life involve improved water conservations abilities and metabolic rate depression in Littorina saxatilis. Marine Ecology Progress Series 224: 171-186.

Stickle B, A Kaper, L Liu, E Gnaiger \& Y Wang. 1989. Metabolic adaptations of several species of crustaceans and molluscs to hypoxia: Tolerance and microcalorimetric studies. The Biological Bulletin 177: 303-312.

Tomanek L \& B Helmuth. 2002. Physiological ecology of rocky intertidal organisms: a synergy of concepts. Integrative and Comparative Biology 42: 771-775.

Widdows J, BL Bayne, DR Livingstone, RI Newell \& $\mathbf{P}$ Donkin. 1979. Physiological and biochemical responses of bivalve mollusks to exposure to air. Comparative Biochemistry and Physiology 62: 301-308.

Zar J. 1996. Biostatistical analysis, 662 pp. Prentice-Hall, New Jersey.

Recibido el 8 de agosto de 2008 y aceptado el 21 de enero de 2009 